\title{
Techno-aesthetic spaces of fiction: Lapsus Trópicus and the Anthropocene dialetic
}

\author{
Karen Aune \\ Universidad de los Andes| Colombia| kaune@uniandes.edu.co
}

\begin{abstract}
This paper is derived from a research through the creative practice, that aims at the conception of an artwork that reflects on the relationship between the human being, technology and nature. This is shown through the creation process of Lapsus Trópicus, an installation whose origin lies in the hypothesis of what would happen if a glitch -a computer error - began to transform a specific ecosystem. The research took into account the conceptual, formal and technical aspects of an artistic process that dialogues with references such as art, design, architecture, science fiction and key historical moments in the development of science.
\end{abstract}

Keywords: Art, Science; Technology; Parametric design; Science fiction

\section{INTRODUCCIÓN}

El presente proyecto parte de una investigación desde la practica creativa, y tiene como objetivo generar una reflexión sobre la relación entre el ser humano, la tecnología y la naturaleza, mediante el proceso de concepción y desarrollo de la obra Lapsus Trópicus, cuyo origen se encuentra en la hipótesis ficticia, sobre qué pasaría si un glitch —un error informático- empezara a transformar un ecosistema específico. La obra llama la atención a las causas y consecuencias de la evolución del ser humano mediante las tecnologías que ha creado, su impacto sobre la naturaleza y las posibles consecuencias de la era del Antropoceno.

Lapsus es la palabra en latín para error o imprevisto, y a la vez glitch, significa lo mismo el ámbito informático. Mientras la palabra tropicus tiene un origen grecolatino que significa vuelta, y también hace referencia a la zona tropical, consecuencia de la combinación de la inclinación del planeta y su vuelta al sol, lo que da como resultado una región que tiene la mayor diversidad ecológica del planeta. De este modo, el título de la obra, Lapsus Trópicus, habla de un glitch en un ecosistema biológico, pero a la vez artificial y virtual en donde los límites entre el ser humano y la naturaleza se diluyen en este universo imaginario.

Este trabajo tiene como objetivo aclarar algunos de los aspectos conceptuales, derivados del proceso creativo llevado a cabo para la concepción de la obra, que dialoga con referencias como el arte, el diseño, la arquitectura, el cine de ciencia ficción y momentos históricos claves en el desarrollo de la ciencia.

\section{METODOLOGÍA}

\section{DE LA PRAXIS ARTISTICA A LA MATRIX}

Esta investigación tiene la obra de arte como su eje central, debido a que "la obra incorpora las respuestas de tal investigación por medio de una expresión simbólica" (Haseman, 2006), y tal obra "produce un conocimiento muy específico mediante la práctica con los materiales, ya que esta induce un cambio en el pensamiento, y que hay una reciprocidad entre la teoría y la praxis" (Bolt, 2007). De esta manera esta investigación desde la practica creativa tiene tres ejes desde los cuales se pueden plantear varios interrogantes que se complementan. El eje teóricoconceptual, que apoya las metáforas visuales de la obra; el eje formal, mediante la creación de elementos estéticos y comunicacionales coherentes al concepto; y el eje técnico que implica la materialización de ideas mediante la experimentación de materiales y tecnologías, considerando que no hay una frontera rígida entre pensamiento y materialización.

La ciencia ficción ha sido un referente importante para la concepción de mi obra debido a su constante especulación sobre el futuro de cada tecnología, los modos en que nos adaptamos a estas y los posibles efectos de su evolución, mediada por la naturaleza humana. En la ciencia ficción es factible explorar las utopías y las distopías mediante la creación de universos únicos, así, hay espacio para lo inimaginable, pero también lo hay para la reflexión sobre temas muy vigentes y necesarios, como anticipar el futuro a través de las acciones del presente. Por esta razón he desarrollado la reinterpretación visual de problemáticas relacionadas con los encuentros entre el tiempo y el espacio, lo virtual y lo real, lo orgánico y lo mecánico, y los acoplamientos entre lo digital y la biología. Técnicamente, he trabajado la apropiación fotográfica y videográfica en las construcciones pictóricas, escultóricas y espaciales de mi obra, valiéndome, entre otros recursos, del diseño y la fabricación digital para resaltar las traducciones del trabajo manual a las tecnologías de reproducción de imágenes: de lo inmaterial a lo material; de crear a replicar; de la mente humana a la inteligencia artificial.

¿Cuál es el lugar del cuerpo o de la tecnología? ¿Cómo interpretar visualmente nuestra experiencia vital en un entorno dominado por tecnologías que nos desterritorializan y nos llevan cada vez más a la virtualidad? ¿Qué tan frágiles, o tan inmersos estamos en este universo paralelo que conquistamos y nos conquista a cada día? ¿En qué lado del Matrix ${ }_{1}$ vivimos finalmente? 


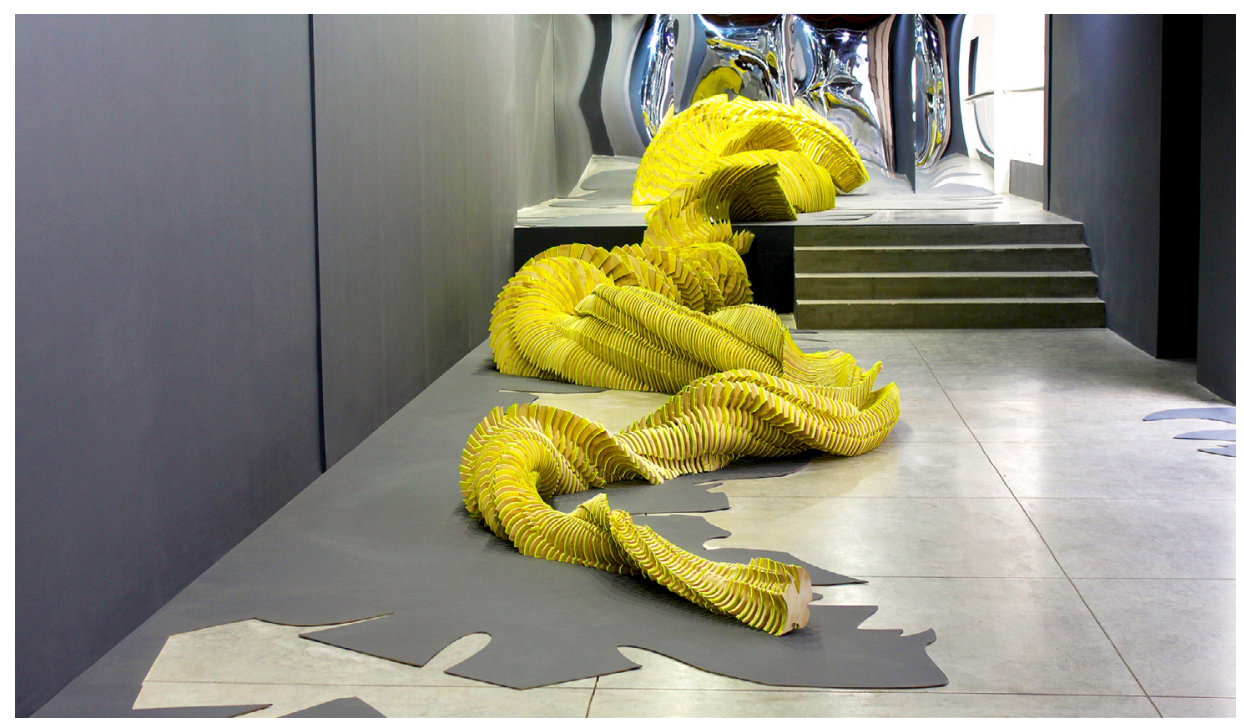

Figura 1: Karen Aune - Lapsus Trópicus

Intervención en espacio de $153 \mathrm{~m} 2$, pintura sobre muro y en cortes de MDF de $3 \mathrm{~mm}$ sobre piso, espejos cóncavos en acrílico de 2 mm sobre muro. Pieza principal: corte numérico en tríplex de 12 mm y acrílico de $3 \mathrm{~mm}-95$ x 182 x $1.600 \mathrm{~cm}$. 2015

\section{RESULTADOS}

\section{LAPSUS TRÓPICUS: LA MUTACIÓN ESPACIO TEMPORAL}

La obra Lapsus Trópicus fue desarrollada para el espacio de la Fundación El Faro de Tiempo en Bogotá en el año 2015, y consistió en una instalación site specific, que estimulaba al espectador a través de los sentidos a reconstruir en su mente un universo alterno y expandido.

Las paredes del espacio se extendían al suelo mediante formas orgánicas, como sombras imaginarias de un lugar infinito y artificial, que al continuar a través de los espejos ligeramente cóncavos ubicados en los extremos de la sala, distorsionaban la imagen de la pieza escultórica principal, fabricada digitalmente en madera triplex y acrílico, que se desplazaba de forma invasiva por el espacio como un organismo vegetal mutante en un constante movimiento visual, que pareciera volver a salir del piso al otro extremo del espacio, a través de una segunda escultura paramétrica que ascendía como una planta en búsqueda de luz.

La experiencia se complementaba mediante una atmósfera sonora 2 , concebida a partir de grabaciones realizadas en florestas tropicales en el momento en que la fauna diurna empezaba a dar lugar a la nocturna y los sonidos a transformarse como consecuencia del cambio lumínico. La grabación se modificó utilizando como base el código genético del Light Harvesting Complexз que funciona como antenas procesadoras de luz en las Monocotiledonia clase botánica de las especies utilizadas para el diseño de la obra-, con el fin de tener una base numérica para modificar el sonido bajo la misma lógica de parámetros de las piezas escultóricas.

Cada elemento formal presente en la obra, responde a un eje comunicacional, el acrílico amarillo lumínico, a la fotosíntesis mediante el plástico; la madera tríplex artificial, a la bioética; la morfología del giro, a la transformación del
ADN; los espejos cóncavos, a la dualidad mutante del doppelgänger5 y su continuidad simétrica en el espacio virtual; el espacio gris como sombra, al espacio infinito de un render, y, por último, el sonido como eco del ADN de las plantas que mutan en tiempo real.

\section{DISCUSIÓN}

\section{LA EXPERIENCIA TECNESTÉTICA}

En los últimos 19 años mi obra empezó a incorporar elementos en los que se podía leer una especie de fusión entre el espacio orgánico y el numérico, lo real y su réplica expandida, como si este fuera la continuación de un universo virtual. Al borrar estos límites, se inicia una conversación sobre quiénes somos y cómo experimentamos o habitamos el planeta. Por un lado, tenemos la búsqueda de lo artificial como el paradigma de la perfección, por el otro, el cuestionamiento sobre la imperfección de la existencia misma.

El concepto de glitch como una falla digital que altera la organización del píxel, siendo este la síntesis de la imagen a partir del cálculo, se complementa con la teoría de la "experiencia tecnestética" planteada por Couchot (2003). El autor reflexiona sobre los cambios sucesivos en la tecnología y cómo estos han modificado los modos de percepción, teniendo en cuenta que las técnicas son parte de un conocimiento complejo del que las opera, llevando siempre su trazo o singularidad. Por consiguiente, este proceso parte de una experiencia esencialmente sensible, donde el autor comparte la subjetividad de la obra con una tecnología que le aporta y la modifica perceptualmente.

En suma, la tecnología no es un mero canal para la solución de un problema creativo, se trata de un elemento vivo con el cual se establece un intercambio, debido a que esta modifica, interfiere y transforma no solo el producto final, sino la misma percepción del artista así como la del espectador. Couchot afirma que las técnicas no son apenas medios de producción sino de percepción, debido 


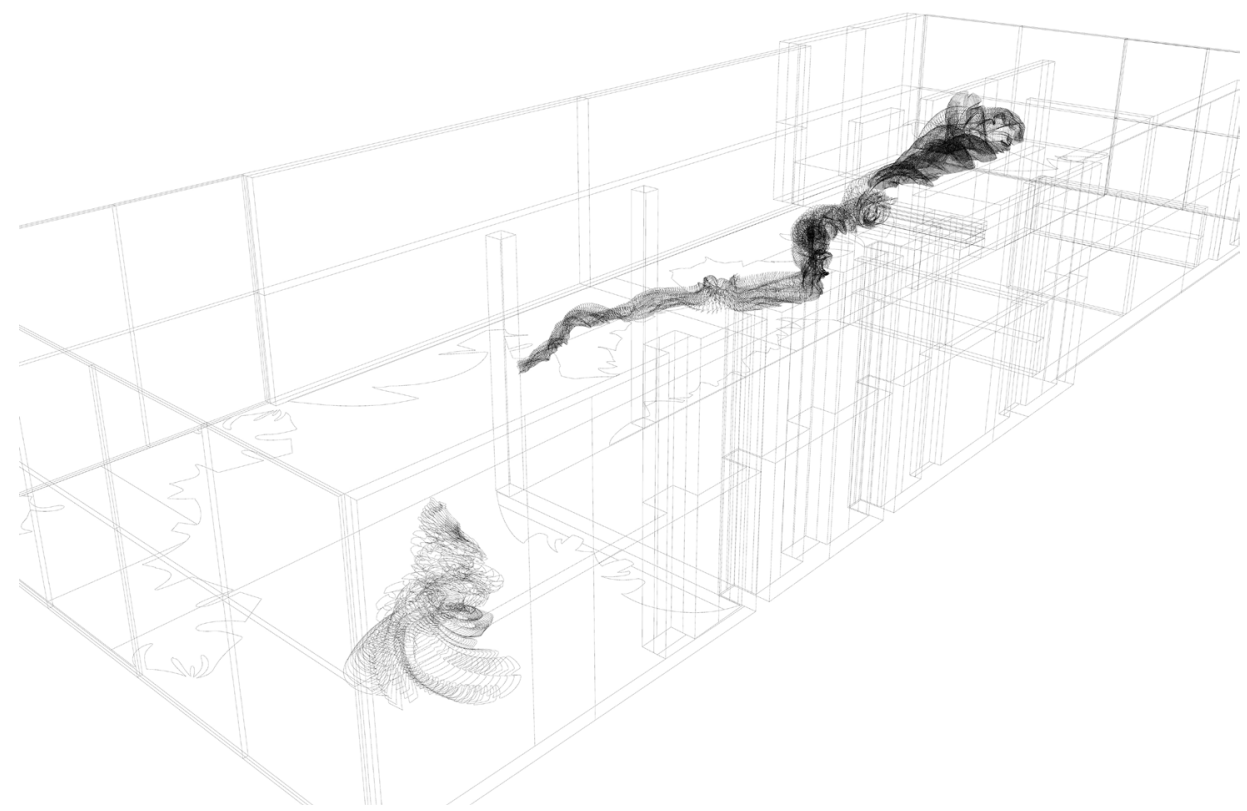

Figura 2: Karen Aune - Lapsus Trópicus: Planos

Impresión digital sobre papel Hahnemühle Photo Matt Fiber 50 x 80 cm. 2015

a que el autor como operador, la controla y la manipula teniendo una experiencia íntima que transforma su percepción del mundo: la experiencia tecnestética. De esta manera, considera la idea del autor como una unidad con la tecnología, un conjunto que evoluciona en la manera de hacer y crear.

Es importante recordar las distintas tecnologías que han sido transformadas a través de los siglos y que ahora hacen parte de las herramientas que utilizamos los artistas. La introducción de artefactos ópticos como apoyo a los procesos de pintura (1420 y 1430) fue un hecho importante que evolucionó hacia la materialización de la primera imagen fotográfica. Según la investigación de Hockney y Falco es posible comprobar cuando se ha usado un artefacto óptico en la realización de una pintura, cuando se identifica el vestigio característico de un error óptico, siendo este el rastro de la impronta de una tecnología que la pone en evidencia.

Los experimentos realizados por Marey (1899) y Muybridge (1872-1873) con el objetivo de hacer visible lo invisible, hicieron posible tener una huella de la esencia del movimiento, al mostrar la relación espacio y tiempo en una única imagen, permitiendo algo que los sentidos humanos no podían percibir. Sus inventos llevaron al desarrollo del cine a partir de toda una evolución de aparatos que vienen cambiar de forma significativa la manera como percibimos el mundo y como creamos a partir de ellos.

Russel A. Kirsch (1957), Ken Knowlton y Leo Harmon (1966), crearon la primera imagen digital procesada de la historia, esta representa la intersección entre la tecnología y el arte en donde la serendipia y la poética, jugaron un papel fundamental en la consolidación de esta alianza que llevó la fotografía al espacio numérico, ampliando sus posibilidades creativas modificando por completo la forma como entendemos la imagen. Igualmente paradójico, es el hecho que la fresadora de control numérico desarrollada por el MIT (1952) en el período de la guerra fría, con e objetivo de hacer más precisa la fabricación de piezas de aviones y artefactos militares, ha basado la evolución de

su desarrollo actual en los sofisticados requerimientos de fabricación de las obras del artista Jeff Koons que está empujando las barreras de la ingeniería como nunca se habia hecho antes, contribuyendo a cambios significativos en la tecnología (Nadya Peek, 2016).

El colectivo multimedia panGenerator realizó Float en 2012, una instalación que consistía en una pecera rodeada de dos cámaras, las cuales rastreaban y traducían los movimientos de un pez contenido en ella para materializar su comportamiento en una impresión 3D. Krystof Golinski (2012), miembro del colectivo, cuenta que "la instalación ofrece un buen ejemplo de cómo la tecnología puede servir para ampliar nuestra percepción de belleza, descubrir capas de realidad que antes eran inalcanzables y proporcionarnos una interfaz entre nuestros sentidos y lo imperceptible". Esto me lleva a la búsqueda de Muybridge y Marey que pretendian hacer "hacer visible lo invisible". Lo que me hace entender la impronta tecnológica de cada época y su evolución con base en el concepto de la experiencia tecnestética sugerida por Couchot.

Un objeto tridimensional puede habitar el mundo virtua mediante renders o animaciones pensadas para existir solo en la pantalla, los modelos arquitectónicos son un ejemplo claro de esto, hoy en día es difícil discernir a simple vista una simulación de un proyecto real. Hay una 


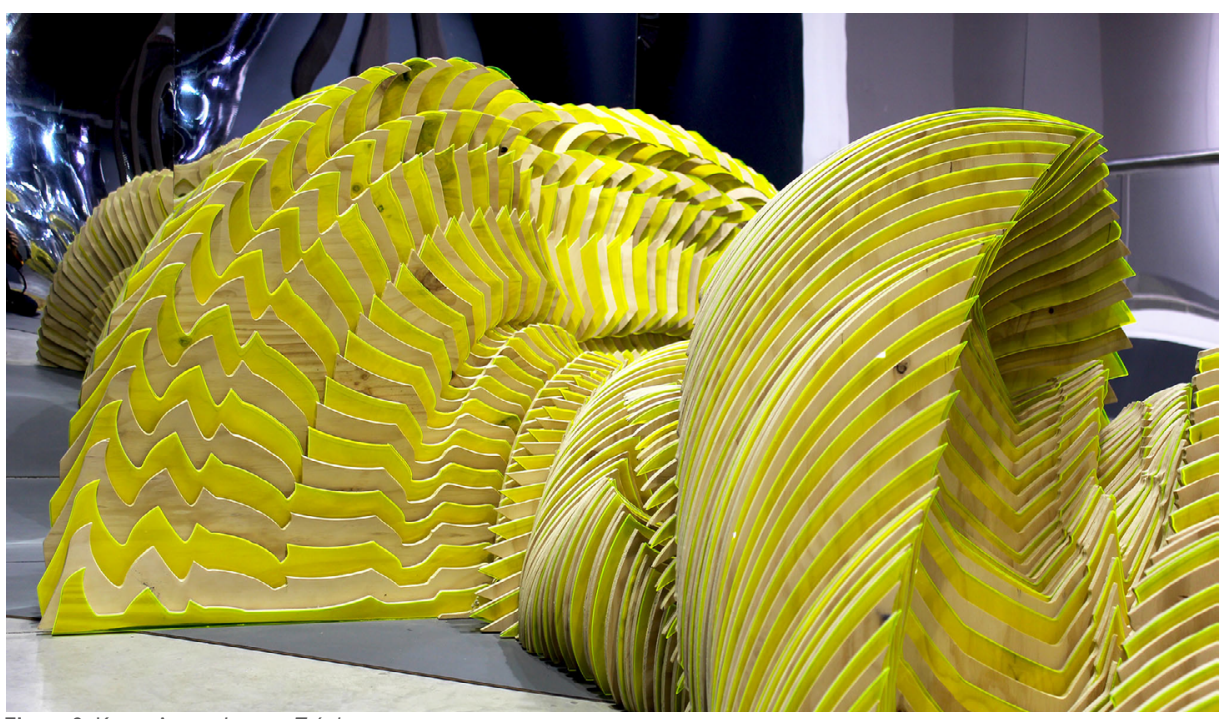

Figura 3: Karen Aune - Lapsus Trópicus

Detalle de la pieza principal: corte numérico en tríplex de $12 \mathrm{~mm}$ y acrílico de $3 \mathrm{~mm}-95$ x 182 × $1.600 \mathrm{~cm} .2015$

cierta belleza en la perfección numérica, quizás por su tangible imposibilidad, por esto, tal vez, estamos modificando todo en nuestro entorno en la búsqueda de este ideal. Todo es un producto recién salido de la máquina que lo crea y lo capitaliza. Las frutas no tienen bichos, las personas no envejecen, las casas no se deterioran, las plantas no se marchitan, los animales son dóciles, las nubes de lluvia no inundan las ciudades inmaculadas y el sol nunca calienta. ¿Todo es perfecto? Finalmente, un mundo sin órganos, sin carne, sin putrefacción, miseria o dolor... Este sería el sueño del cyborg, o la pesadilla propuesta por Critical Art Ensamble (2001) "(CsO) el cuerpo sin órganos sueña con un cuerpo que jamás existió".

Los inventos mencionados transformaron radicalmente la historia de las imágenes y de los objetos, y aunque sus propósitos fueran inicialmente alejados del arte, la manera como se manifestaron estas tecnologías no solo ejerció una gran influencia en los artistas, sino que dejó en evidencia su impronta en las obras mediadas por ellas, y esta fue más que un rastro técnico, sino que un statement de una época impregnado de sus valores.

A lo largo de los años, distintas herramientas tecnológicas complementaron significativamente mi proceso creativo. Por esta razón entendí que el proceso de elaboración de mi obra constituye una gran parte de mi propia experiencia tecnestética, que en colaboración con dicha tecnología y su impronta, se extiende al espectador que, a su vez, es capaz de descifrar de forma poética los códigos estéticos implícitos en el objeto creado.

\section{LA TECNOLOGÍA DE LA MENTE}

La bióloga colombiana Brigitte Baptiste plantea de manera muy sensible, sobre la relación que tenemos, con el medio ambiente y las plantas como mediadoras de la acción colectiva. Ella reflexionó sobre el conocimiento del yagé 6 , entre otros enteógenos 7 , utilizados por distintas culturas indígenas y se refirió a este como: una "tecnología de la mente", un invento increíble por sus propiedades neurológicas, una tecnología para la gestión ambiental que parte del cuerpo, debido a que se trata de una sustancia que disuelve el ego.

Baptiste aclara que la cultura del vicio, realmente, es la adicción al alimento continuo del ego, como por ejemplo, la legitimación de las relaciones sociales, y la necesidad de probar el éxito y la felicidad constantemente, como la noción de triunfo desde un ego desbordado. Para Baptiste (2011), estas "tecnologías de la mente", promueven la cooperación y la interacción entre las personas, además de una comprensión del entorno.

Los modos de percepción, al ser alterados mediante la experiencia en los espacios creados por algunos artistas, pueden ser comparables a una experiencia enteógena, la cual nos conecta con el entorno, ampliando nuestra noción de ser y estar, a una noción colectiva del pertenecer y existir como parte de un sistema complejo y sofisticado: la naturaleza.

Algunos artistas tienen la particularidad de que sus obras se presentan de forma experiencial, al hackear los sentidos y la percepción de un espectador que, al entrar, experimentar y participar de la pieza instalativa, comprende de forma emocional y corporal los aspectos comunicacionales de tales piezas. De esta manera, se aprende a través de los sentidos y no de la razón.

En su más reciente obra, el artista brasileño Ernesto Neto generó una reflexión sobre el conocimiento ancestral de distintas comunidades indígenas que se relacionan con el entorno de una manera simbiótica, y cómo estos nos pueden ayudar a recuperar la conexión perdida con el todo, para entendernos como parte de un contexto y no como un espectador de la vida, siendo esta una experiencia compleja. Neto llevó a la Bienal de Venecia del 2017 la obra Un sagrado lugar, que consistió en la recreación de un Cupixawa, una tienda ceremonial de los indígenas huni kuin, habitantes de la selva amazónica brasileña en el estado de Acre. La obra consistía en una 
experiencia, lo que suele ser característico del trabajo de Neto; sin embargo, en esta intervención particular, el asunto trascendía fronteras, en tanto los espectadores eran invitados a participar de los rituales de aprendizaje, sanación y conexión con la naturaleza practicados por los huni kuin, mediante cantos ceremoniales y el uso de la ayahuasca. En una entrevista, Neto dice que nos estamos alejando de la naturaleza y nos estamos enfermando, que no entendemos que esta no es algo externo, sino que hacemos parte de ella. En efecto, somos naturaleza.

De igual forma, el artista brasileño Henrique Oliveira hace parte de esa generación encargada de aportar nuevos elementos a la propuesta de Helio Oiticica y Lygia Clark, y, a su vez, de dar una continuación transversal a la obra de Ernesto Neto. Oliveira declara que parte del universo pictórico, de la experimentación con la materia, la cual encuentra en los tapumes - planchas de tríplex de baja calidad, utilizados en Brasil para tapar provisionalmente espacios donde hay una construcción en proceso-. Los tapumes son la materia prima de la obra de Oliveira, quien los maneja con una plasticidad impresionante, como si fueran brochazos de pintura, para construir ambientes tridimensionales que dialogan y se expanden en el espacio. Sus instalaciones dan al espectador la posibilidad de una exploración sensorial compleja, desde el olor a la madera vieja, hasta la multiplicidad de colores táctiles presentes en el interior y en el exterior de los espacios creados. De esta forma, la obra propone una metamorfosis que, a partir de los tapumes encontrados en los espacios urbanos, se desplaza hacia su origen, el árbol. Árbol que a la vez es y toma la arquitectura del espacio de forma voraz e invasiva y a través de un constante movimiento que parece retomar lo que es suyo. Pasear adentro de estas raíces y troncos es sentirlos vivos, expandiéndose cada segundo.

Por otro lado, me parece importante resaltar la forma de manejo del espacio del artista danés de ascendencia islandesa Olafur Eliasson. Creador de obras que apelan a los sentidos como forma de generar colectividad, Eliasson busca que el espectador experimente la obra desde su inteligencia emocional y sensorial, para lo cual recurre a los fenómenos naturales como parte importante del concepto de su trabajo. Al igual que Neto y Oliveira, Eliasson guía al participante de la obra mediante los sentidos, encargados de generar sentimientos que, a su vez, generan conocimiento. La emoción es un gran canal de conversación y la participación es el medio para generar el diálogo.

Este artista interdisciplinar es absolutamente coherente en todos sus proyectos, los cuales tienden hilos entre arte, diseño y arquitectura, de forma tan espectacular como sensible. En el Estudio Olafur Eliasson se han gestado proyectos de diferentes índoles, mediante la colaboración de un equipo de más de 100 personas. Tales proyectos van desde la creación de la fachada estructural del Harpa Concert Hall en Reykjavik (2011), hasta un libro de recetas vegetarianas, en el cual se expresa la importancia del cocinar, comer y crear en comunidad, o el proyecto Little Sun que, desde el 2012 lleva luz mediante un pequeño artefacto captador de energía solar a comunidades que aún no tiene acceso a este recurso.

En el año 2003, Eliasson realizó The Weather Project, intervención que tuvo lugar en la Turbine Hall de la Tate Modern de Londres. En esa ocasión, el artista instaló la mitad de un sol, realizado con lámparas de monofrecuencia, que reflejado en el techo cubierto de espejos no solo completaba su circunferencia sino que transformaba las dimensiones del ambiente, incitando la participación de las personas que se encontraban en el lugar. Espacio en el cual se disipaba una especie de niebla, lo que creaba un hermoso paisaje en el ambiente, mediante un efecto duotono. Además de bellísima, la intervención generaba un sentido de colectividad y participación. Para este artista es importante que se pueda vivir y experimentar la obra en comunidad, sin necesariamente estar de acuerdo o entender algo de la misma manera, pensamiento —-bastante escandinavoque está siempre presente en su obra. Eliasson, de forma similar a Ernesto Neto, cree en la experiencia a través de los sentidos y las emociones, así como en el gesto corporal, como una forma de entender y aprender un mensaje o captar una idea poética.

Radicado en Canadá, el arquitecto inglés Philip Beesley realiza un trabajo experimental que atraviesa y trasciende nociones de la arquitectura, el arte digital, la tecnología, el diseño, la biología, la moda y la ingeniería. Desde hace varios años, Beesley trabaja con un equipo de investigación multidisciplinar llamado Living Architecture Systems Group en la Universidad de Waterloo en Canadá. Su proyecto consiste en crear arquitecturas sensibles o responsivas, por medio de ambientes interactivos desarrollados de forma que se asimilen a ecosistemas con la capacidad de moverse, responder y aprender, con sistemas metabólicos que puedan renovar sus entornos, de forma adaptativa y empática hacia sus habitantes. Según Beesley (2012), su investigación se plantea como una arquitectura viva y puede integrar comportamientos basados en máquinas e intercambios químicos, además de tratar de entender las respuestas cognitivas, fisiológicas y emocionales de los habitantes. A largo plazo, se trata de encontrar estrategias prácticas para lograr la resiliencia y la adaptabilidad en estados de desequilibrio, como los que ocurren actualmente en el entorno natural, preparando una nueva generación de diseñadores con habilidades críticas y capacidad para trabajar con ambientes complejos.

Si la arquitectura de Beesley pretende aprender de la naturaleza para comunicarse con nosotros, la arquitectura, Neri Oxman, profesora de artes y ciencias de medios del MIT, muestra cómo esta naturaleza puede enseñarnos a trascender nuestra existencia actual. En varias de sus conferencias, ella afirma: "en la naturaleza no encontramos ensambles", todo está formado mediante una variación inteligente de la misma materia. Su trabajo busca unir dos perspectivas, la tecnología y la naturaleza, mediante un proceso que se aleje del ensamble y se acerque al crecimiento. Oxman acuñó el término material ecology, que, en sus palabras, "considera que la computación, la fabricación y el material en sí, son dimensiones inseparables del diseño". La arquitecta tiene, entonces, como objetivo aumentar la relación entre el diseño y los entornos naturales mediante principios inspirados y diseñados por la naturaleza, a través de nuevas tecnologías de fabricación y construcción digital que van de microescalas a macroescalas.

Los proyectos que desarrolla Oxman entienden los procesos biológicos como una tecnología sofisticada de la cual debemos aprender y que nos lleva a reflexionar sobre nuestros propios procesos de vida. En el 2013, con su grupo de investigación en el MIT, Mediated Matter, realizó un pabellón encargado de explorar la relación entre la fabricación digital y la biológica desde un producto de escala arquitectónica. Primero, el grupo realizó estudios sobre la capacidad del gusano de seda para generar un capullo tridimensional a partir de un único hilo, indagando 


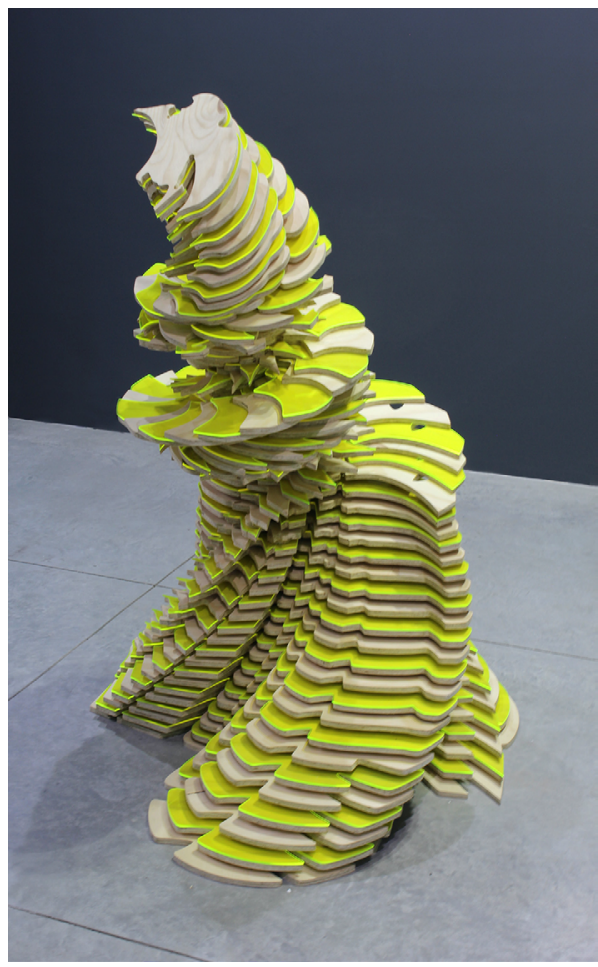

Figura 4: Karen Aune - Lapsus Trópicus: Pieza secundaria Corte numérico en tríplex de $12 \mathrm{~mm}$ y acrílico de $3 \mathrm{~mm}$ $92 \times 86 \times 120 \mathrm{~cm} .2015$

en los patrones de construcción. Una vez finalizadas las inquisiciones preliminares, la investigación procedió a utilizar un algoritmo para construir la estructura hexagonal del pabellón, con el fin de que un conjunto de 6.500 gusanos la pudiera tejer de forma plana, y además, llegar a su proceso de metamorfosis natural. Finalmente, instalaron esta estructura en un espacio con condiciones de luz y sombra que pudieran informar sobre el comportamiento de los gusanos y la densidad de su tejido, conforme los ciclos de luminosidad. De esta manera, el resultado fue un bellísimo pabellón tejido por estas pequeñas criaturas. Además, el experimento generó una profunda reflexión sobre los procesos de la naturaleza y cómo debemos aprender más de ellos.

Los artistas, arquitectos y diseñadores a los cuales me refiero tienen en común el sentido participativo e interdisciplinar en sus proyectos, sus obras son informadas por la naturaleza, algunas son responsivas y empáticas como si fueran vivas, otras buscan alterar los sentidos mediante la percepción. Estos proyectos son elaborados de una manera tal que consiguen borrar el límite entre obra y espectador, con el objetivo de que este último entienda que es una parte importante de ella, específicamente, al complementar el sentido de los múltiples mensajes que conforman casi una conversación privada con cada uno de los individuos que habita o recorre la obra, aun cuando esta relación suceda en un espacio colectivo.

De esta manera, considero que mi trabajo a lo largo de los años se ha enmarcado en una búsqueda similar. Las piezas que he mencionado son instalaciones y estas tienen ciclos de vida, se transforman según el nuevo lugar en el cual ocasionalmente vuelven a existir, a veces desaparecen por completo y la memoria parece apenas quedar en el registro visual tomado. Es interesante pensar la magnitud de estos proyectos y la vida corta que tienen en el sentido material, no obstante la vida larga que tienen en la memoria de aquellos que los experimentaron, en un proceso de aprendizaje completamente novedoso y particular.

\section{CONCLUSIONES}

La reflexión realizada durante el proceso creativo para la realización de Lapsus Trópicus, me hizo entender la conexión entre las obras que he realizado en los últimos años y su pregunta implícita: cómo hemos evolucionado mediante nuestros inventos, cómo estos nos han transformado como seres humanos y, en consecuencia, cómo hemos cambiado nuestro entorno.

Un concepto similar lo vengo a encontrar en la película de ciencia ficción: Annihilation del director Alex Garland (2018), basada en la novela homónima de Jeff VanderMeer (2014), que especula sobre la capacidad autodestructiva del ser humano al crear una metáfora sobre la interconectividad entre todos los elementos presentes en un ecosistema, mediante un fenómeno que refracta el código genético de sus habitantes y los muta entre si. El término annihilation puede significar erradicación o extinción, pero también la rendición del yo en un sentido místico, y desde la física, es la conversión de la materia en energía.

La materialización de la obra, además de ser un problema técnico o práctico, conllevaba a problemáticas y soluciones conceptuales. Consecuentemente, este proceso de investigación mediado por la práctica creativa, me llevó a reflexionar sobre cómo la tecnología que construye y a la vez modifica una obra mediante su rastro, va mucho más allá, en el sentido en que esta también modifica lo que somos en términos éticos y filosóficos.

Mi búsqueda en los distintos recursos tecnológicos para resolver la obra, como el diseño y la fabricación digital, viene de la intención de hacer presente el concepto de "la experiencia tecnestética" sugerida por Couchot (2003), "donde autor y tecnología se complementan", lo cual deja en evidencia las posibilidades y límites de esta tecnología implícita en la elaboración de casi todas las cosas que experimentamos en la actualidad.

Esto se da con el motivo de abrir la discusión sobre las posibilidades presentes en la unión entre ser humano, tecnología y naturaleza, explorando los debates sobre biotecnología y mundos virtuales versus la fragilidad del universo biológico, apuntando y cuestionando la ficción de un futuro por venir. De esta manera, es posible entender lo que afirma Neri Oxman (2015), cuando dice que "en la naturaleza no hay ensambles, sino una continuidad inteligente en la materia, de modo tal que la tecnología debería ser utilizada para crear diseños biológicamente informados por, con y para la naturaleza".

La metáfora propuesta por Brigitte Baptiste (2011) es muy poderosa al definir el yagé como una "tecnología de la mente", que al amplificar la percepción diluye el ego y nos conecta con el entorno. Tal tecnología dejaría, mediante una nueva experiencia tecnestética, una distinta impronta sobre nuestro entorno, que nos modificaría y nos reconectaría con los ecosistemas mediante nuestros sentidos, al recordarnos que somos parte de un mundo 
real y responsables por nuestra propia sintropías, la reorganización y el equilibrio. Si permitimos a nuestros cuerpos electrónicos disolverse en la Matrix del ego deseante, lo único que nos quedaría sería la entropía, la desorganización sin regreso.

Así que, Lapsus Trópicus busca hackear los sentidos con el fin de que entendamos de una manera poética el impacto que tenemos sobre el medio ambiente, recordándonos que somos parte de ello, y no meramente un espectador que racionaliza sus posibilidades 0 utilidades desde los extraños valores de la era del Antropoceno, la actual era geológica que ha sufrido el impacto de las actividades de los seres humanos sobre los ecosistemas.

La pandemia es la consecuencia de la intervención bruta del ser humano en un delicado ecosistema. Los efectos del cambio climático serán más devastadores que la pandemia. La violencia que el ser humano ejerce contra la naturaleza se está volviendo contra él con más fuerza. En eso consiste la dialéctica del Antropoceno: en la llamada Era del Ser Humano, el ser humano está más amenazado que nunca. (Han, 2020)

En suma, Lapsus Trópicus se materializa como una floresta digital, un ecosistema transformado por la tecnología; un mundo artificial, sensorialmente virtual, donde es importante la idea de lo fractal; un espacio fragmentado, simbiótico, orgánico e invasivo que proporciona la inmersión del espectador y a la vez le da la sensación de ser un explorador en un mundo por venir. Un territorio alterado por un glitch, generado desde su propio medio de producción en el cual cada elemento botánico es clonado y muta digitalmente una y otra vez hacia otro elemento más complejo. Esta floresta pretende ser la matrix entre un mundo virtual y su simulacro: el biológicamente real.

\section{CITAS}

1. La utilización de la palabra matrix en el texto es una metáfora para pensar el espacio virtual desde el punto de vista de la película The Matrix que es una trilogía de películas de ciencia ficción dirigida por las hermanas Wachowski (1999-2003), que narra un mundo dominado por las máquinas e inteligencias artificiales que se alimentan de la conectividad de los humanos que viven en una ilusión colectiva inmersos un espacio virtual conocido como Matrix (The Matrix, s. f.).

2. Diseño de sonido en colaboración con Alejandro Zuluaga y Pol Moreno.

3. Light Harvesting Complex es un complejo de pigmento y proteína que recolecta energía de la luz y la convierte en energía de excitón que puede migrar al centro de reacción fotosintética donde se produce la fotosíntesis. La luz es absorbida por las moléculas de pigmento que están unidas a la proteína (Light Harvesting Complex, s. f.).

4. Monocotiledóneas es una de las dos clases de plantas con flor (angiospermas). Son casi siempre herbáceas y a ella pertenecen plantas muy conocidas, como lirios, azucenas, orquídeas, gramíneas (trigo, maíz, caña de azúcar) y palmeras (s. f.).

5. La palabra doppelgänger significa "doble andante" en alemán, el término se utiliza para designar a cualquier doble (gemelo) de una persona o al fenómeno de la bilocación. El término es común en obras de ciencia ficción, en forma de cibergemelos, bots (programas informáticos que imitan nuestro comportamiento), en este caso podría ser nuestro rastro de información virtual (Krotoski, 2016).

6. La ayahuasca o yagé, es una mezcla de dos plantas: la enredadera de ayahuasca (Banisteriopsis caapi) y un arbusto llamado chacruna (Psychotria viridis), que contiene el alucinógeno dimetiltriptamina (DMT). Es utilizada desde hace más de 5.000 años por los chamanes o curanderos del Amazonas como un camino para obtener la expansión de la conciencia (¿Qué es ayahuasca?, s.f.).

7. Un enteógeno es una sustancia vegetal utilizado en contextos ritualísticos y chamánicos. Tiene propiedades psicotrópicas y puede causar un estado modificado de conciencia. Su efecto es el de trasladarnos a una realidad, que se percibe más auténtica que el mundo habitual, una dimensión cargada de profundo significado religioso e impregnada de un sentimiento de lo sobrenatural (Wagner, 2010).

8. El matemático italiano Luigi Fantappiè, que trabajó con Einstein, descubrió que la solución causal se rige por la ley de la entropia, mientras que la solución retrocausal se rige por una ley simétrica la cual denominó sintropía, combinando las palabras griegas syn que significa convergencia, y tropos, que significa tendencia. La entropía implica la tendencia de la energía hacia la disipación, también conocida como la ley de la muerte térmica o el desorden. Por el contrario, la sintropía implica la tendencia a concentrarse y absorber energía, el aumento de las temperaturas, la diferenciación, la complejidad, la formación de estructuras y organizaciones. La ley de la sintropía explica las sincronicidades y cómo estas pueden considerarse la causalidad fundamental que gobierna la vida (Di Corpo, 2015).

\section{REFERENCIAS}

Adam, H. C. (2010). Eadweard Muybridge: The Human and Animal Locomotion Photographs. Köln: Taschen.

Annihilation. IMDB (s. f.). Recuperado de https://www.imdb.com/title/tt2798920/

Baptiste, B. (2011). El jaguar y la telepatina del yagé - Amazonía Perdida. Parque Explora. Recuperado de https://www.youtube.com/watch?v=gK3BWngw7ol

Baudrillard, J. (2002). Cultura y simulacro. Barcelona: Kairós.

Williams, J. H. (2008). Employee engagement: Improving anticipation in safety. Professional Safety, 53(12), 40-45

Baudrillard, J. (1999). Tela total. Porto Alegre: Sulina.

Beesley, P. (s. f.). Recuperado de http://philipbeesleyarchitect.com/

Bolt, B. (2007). The magic is in handling, en E. Barret and Bolt (ed.), Practice as Reserach: Aproaches to Creative Arts Enquiry, London and New York: I.B. Tauris

Couchot, E. (2003). A tecnología na arte da fotografía à realidade virtual. Porto Alegre: Editora da UFRGS.

Critical Art Ensenble. (2001). Disturbio Eletrônico. São Paulo: Conrad.

Di Corpo, U., y Vannini, A. (2015). Syntropy: The Spirit of Love. New Jersey: ICRL Press.

Engberg-Pedersen, A. (ed.). (2012). Studio Olafur Eliason: An Encyclopedia. Köln: Taschen $\mathrm{GmbH}$.

Gómez, M. (2017). Historia(s) de la imagen digital. Interartive. Recuperado de https://interartive.org/2017/04/historias-de-laimagen-digital-marisa-gomez

Haseman, B. (2006), A manifesto for perfomative research, Media International Australia Incorporating Culture and Policy. Recuperado de http://www.emsah.uq.edu.au/mia/issues/miacpll8.htm

Henrique Oliveira (s. f.). Recuperado de http://www.henriqueoliveira.com

Hockney, D. (2001). El conocimiento secreto: el descubrimiento de las técnicas perdidas de los grandes maestros. Barcelona: Ediciones Destino.

Knowlton, K. C. (2001). On the Frustrations of Collaborating with Artists. Ken Knowlton. Recuperado de http://dada.compart-bremen.de/item/agent/203 
Knowlton, K. C. (2004). Portrait of the Artist as a Young Scientist by Ken Knowton. Digital Art Guild. Recuperado de http://www.digitalartguild.com/content/view/26/26/

Krotoski, A. (2016). ¿Qué es un doppelgänger y dónde está el tuyo? BBC Mundo. Recuperado de https://www.bbc.com/mundo/noticias/2016/05/160427_doppe Igangers_gemelos_extranos_fantasmagoricos_selfies_finde_ dv

Ernesto Neto Venice Biennale 2017. Judith Benhamou-Huet Reports. (2017). Recuperado de https://www.youtube.com/watch?v=A_3FqcP_18

Oxman, N. (n.d.). Recuperado de http://www.materialecology.com/neri-oxman

Oxman, N. (2015). Diseño en la intersección de la tecnología y la biologia. TED Talks. Recuperado de https://www.ted.com/talks/neri_oxman_design_at_the_interse ction of technology and biology?language $=\mathrm{es}$

Pangenerator. (s. f.). Recuperado de http://pangenerator.com

Peek, N. (2016). Historia y aplicaciones de la fabricación digital con Nadya Peek y Manuel Martínez Torá. La hora maker. Recuerado de http://lahoramaker.com/2016/11/07//hm-056historia-y-aplicaciones-de-la-fabricacion-digital-con-nadyapeek-y-manuel-martinez-toran/
Rendules, C. (2020). Byung-Chul Han: El dataísmo es una forma pornográfica de conocimiento que anula el pensamiento. Recuperado de:

https://elpais.com/cultura/2020/05/15/babelia/1589532672 5 74169.html

Studio Olafur Eliasson. (s. f.). Recuperado de http://www.olafureliasson.net

The Science of Digital Fabrication.mit. (2013). Recuperado de http://cba.mit.edu/events/13.03.scifab/

The Matrix. IMDB (s. f.). Recuperado de https://www.imdb.com/title/tt0133093/

Towers, M. Muybridge y Marey. Mariana Phone Home. (s. f.). Recuperado de https://marianaphonehome.wordpress.com/2014/02/02/mu ybridge-y-marey/

Vander-Meer, J. (2014). Books: Best of 2014 Lists Galore. Jeff Vander-Meer. Recuperado de http://www.jeffvandermeer.com/2014/12/

Vannini, A. (2005). Entropy and Syntropy: From Mechanical to Life Science. Neuroquantology. Recuperado de https://www.neuroquantology.com/index.php/journal/article/ view/64

Zampaglione, F., y Jules Marey, E. (s. f.). Proyecto Movimiento Humano. Recuperado de http://proyectomovimientohumano.zampaglione.com.ar/etie nne-jules-marey-1830-1904 